\title{
Analysis of Mixed Methods Using Mesh Dependent Norms*
}

\author{
By I. Babuška, J. Osborn and J. Pitkäranta
}

\begin{abstract}
This paper analyzes mixed methods for the biharmonic problem by means of new families of mesh dependent norms which are introduced and studied. More specifically, several mixed methods are shown to be stable with respect to these norms and, as a consequence, error estimates are obtained in a simple and direct manner.
\end{abstract}

1. Introduction. In [5] Brezzi studied Ritz-Galerkin approximation of saddlepoint problems arising in connection with Lagrange multipliers. These problems have the form:

Given $f \in V^{\prime}$ and $g \in W^{\prime}$, find $(u, \psi) \in V \times W$ satisfying

$$
\left\{\begin{aligned}
a(u, v)+b(v, \psi) & =(f, v) \quad \forall v \in V, \\
b(u, \varphi) & =(g, \varphi) \quad \forall \varphi \in W,
\end{aligned}\right.
$$

where $V$ and $W$ are real Hilbert spaces and $a(\cdot, \cdot)$ and $b(\cdot, \cdot)$ are bounded bilinear forms on $V \times V$ and $V \times W$, respectively.

Given finite-dimensional spaces $V_{h} \subset V$ and $W_{h} \subset W$, indexed by the parameter $0<h<1$, the Ritz-Galerkin approximation $\left(u_{h}, \psi_{h}\right)$ to $(u, \psi)$ is defined as the solution of the problem:

Find $\left(u_{h}, \psi_{h}\right) \in V_{h} \times W_{h}$ satisfying

$$
\left\{\begin{aligned}
a\left(u_{h}, v\right)+b\left(v, \psi_{h}\right) & =(f, v) \quad \forall v \in V_{h}, \\
b\left(u_{h}, \varphi\right) & =(g, \varphi) \quad \forall \varphi \in W_{h} .
\end{aligned}\right.
$$

The major assumptions in Brezzi's results are

$$
\sup _{v \in Z_{h}} \frac{|a(u, v)|}{\|v\|_{V}} \geqslant \gamma_{0}\|u\|_{V} \quad \forall u \in Z_{h} \text { and } \forall h,
$$

where $\gamma_{0}>0$ is independent of $h$, and $Z_{h}=\left\{v \in V_{h}: b(v, \varphi)=0 \forall \varphi \in W_{h}\right\}$, and

$$
\sup _{v \in V_{h}} \frac{|b(v, \varphi)|}{\|v\|_{v}} \geqslant k_{0}\|\varphi\|_{w} \quad \forall \varphi \in W_{h} \text { and } \forall h \text {, }
$$

Received October 2, 1979.

1980 Mathematics Subject Classification. Primary 65N15, 65N30.

Key words and phrases. Mixed methods, error estimates, stability.

* Sponsored by the United States Army under Contract No. DAAG29-75-C-0024. This material is based upon work supported by the National Science Foundation under Grant MCS7802851 and the Department of Energy under Contract No. E (40-1)3443. 
where $k_{0}>0$ is independent of $h$. Using (1.3) and (1.4), Brezzi proves the following error estimate for the approximation method determined by (1.2):

(1.5) $\left\|u-u_{h}\right\|_{v}+\left\|\psi-\psi_{h}\right\|_{w} \leqslant C\left(\inf _{\chi \in V_{h}}\|u-\chi\|_{v}+\inf _{\eta \in W_{h}}\|\psi-\eta\|_{\omega}\right) \forall h$, where $C$ is independent of $h$.

In [1], [2] Babuška studied Ritz-Galerkin approximation of general, variationally posed problems. The main result of [1], [2], as applied to (1.1) and (1.2), is that (1.5) holds provided

$$
\begin{aligned}
\sup _{(v, \varphi) \in V_{h} \times W_{h}} \frac{|a(u, v)+b(v, \psi)+b(\eta, \varphi)|}{\|v\|_{v}+\|\varphi\|_{\omega}} \geqslant \tau_{0}\left(\|u\|_{v}+\|\psi\|_{w}\right) \\
\\
\forall(u, \psi) \in V_{h} \times W_{h} \text { and } \forall h,
\end{aligned}
$$

where $\tau_{0}>0$ is independent of $h$. It is clear from [1], [2] that (1.3) and (1.4) hold if and only if (1.6) holds. (1.3)-(1.4) or, equivalently, (1.6) is referred to as the stability condition for this approximation method.

The results of [1], [2], [5] can be viewed as a strategy for analyzing such approximation methods: the approximation method is characterized by certain bilinear forms, norms (spaces), and families of finite-dimensional approximation spaces, and if the method can be shown to be stable with respect to the chosen norms, then the error estimates in these norms follow directly, provided the bilinear forms are bounded and the approximation properties of $V_{h}$ and $W_{h}$ are known in these norms. These results can be used to analyze, for example, certain hybrid methods for the biharmonic problem [5], [6]. The results of [1], [2] have also been used to analyze a variety of variationally posed problems that do not have the form (1.1).

There are other problems of a similar nature, however, where attempts at using the results of [1], [2], [5] were not entirely successful since not all of the hypotheses were satisfied: specifically, the Brezzi condition (1.3) or, equivalently, the Babuška condition (1.6), is not satisfied with the usual choice of norms, i.e., the approximation methods for these problems are not stable with respect to the usual choice of norms. This is the case, for example, in the analysis [7] of the HerrmannMiyoshi [15], [16], [20] mixed method for the biharmonic problem. In the analysis of this method, a natural choice for both $\|\cdot\|_{v}$ and $\|\cdot\|_{w}$ is the 1st order Sobolev norm; however, this method is not stable with respect to this choice of norms. As a result of this difficulty, the error estimates obtained in [5] are not optimal. A similar difficulty arises in the analysis of the Herrmann-Johnson [15], [16], [17] and Ciarlet-Raviart [9] mixed methods for the biharmonic problem. In later work of Scholz [23] and Rannacher [22], optimal error estimates were obtained for the mixed methods of Ciarlet-Raviart and Herrmann-Miyoshi. In a forthcoming paper, Falk-Osborn [12] develop abstract results from which optimal error estimates for these and other problems can be derived. However, in neither the work of Scholz 
[23], Rannacher [22], nor Falk-Osborn [12] is the systematic approach of Brezzi and Babuška used.

It is the purpose of this paper to analyze mixed methods for the biharmonic problem by means of the results of Brezzi and Babuška. This is done by introducing a new family of (mesh dependent) norms with respect to which the above-mentioned mixed methods (Ciarlet-Raviart, Herrmann-Miyoshi, Herrmann-Johnson) are stable. Once the stability condition has been checked and the approximation properties of the subspaces $V_{h}$ and $W_{h}$ have been determined in these new norms, the error estimates in these norms follow immediately from the abstract results of Brezzi and Babuška. Error estimates in the more standard norms are then obtained by using the usual duality argument. The results of this paper were announced in [21]. We also note that the methods employed in this paper have been applied to two-point boundary value problems in [3].

Section 2 contains a review of the convergence results of Brezzi and Babuška. In Section 3 we introduce and study the mesh dependent norms and spaces used in the analysis in this paper. In Section 4 we treat three examples previously analyzed in the literature and show how error estimates can be derived from the abstract results in Section 2, used in conjunction with the mesh dependent norms introduced in Section 3. These examples are all mixed methods for the biharmonic problem. The error estimates in the standard norms that are obtained in the present paper and those obtained in [12], using different techniques, are the same.

Throughout this paper we will use the Sobolev spaces $H^{m}=H^{m}(\Omega)$, where $\Omega$ is a convex polygon in the plane and $m$ is a nonnegative integer. On these spaces we have the seminorms and norms

$$
|v|_{m}=|v|_{m, \Omega}=\left(\sum_{|\alpha|=m} \int_{\Omega}\left|D^{\alpha} V\right|^{2} d x\right)^{1 / 2}
$$

and

$$
\|v\|_{m}=\|v\|_{m, \Omega}=\left(\sum_{|\alpha| \leqslant m} \int_{\Omega}\left|D^{\alpha} V\right|^{2} d x\right)^{1 / 2} .
$$

$H_{0}^{m}(\Omega)$ denotes the subspace of $H^{m}(\Omega)$ of functions vanishing together with their first $m-1$ normal derivatives on $\Gamma=\partial \Omega$. We also use the spaces $H^{-m}(\Omega)=$ $\left(H_{0}^{m}(\Omega)\right)^{\prime}$ (the dual space of $H_{0}^{m}(\Omega)$ ) with the norm on $H^{-m}(\Omega)$ taken to be the usual dual norm.

2. Abstract Convergence Results. In this section we review certain results on the approximate solution of saddle-point problems.

Let $V_{h}$ and $W_{h}$ be real Hilbert spaces (indexed by the parameter $h$, where $0<$ $h<1)$ with norms $\|\cdot\|_{V_{h}}$ and $\|\cdot\|_{\omega_{h}}$, respectively, and let $a_{h}(\cdot, \cdot)$ and $b_{h}(\cdot, \cdot)$ be bilinear forms on $V_{h} \times V_{h}$ and $V_{h} \times W_{h}$, respectively. We suppose

$$
\left|a_{h}(u, v)\right| \leqslant K_{1}\|u\|_{V_{h}}\|v\|_{V_{h}} \quad \forall u, v \in V_{h},
$$




$$
\left|b_{h}(u, \varphi)\right| \leqslant K_{2}\|u\|_{V_{h}}\|\varphi\|_{w_{h}} \quad \forall u \in V_{h}, \quad \forall \varphi \in W_{h},
$$

where $K_{1}$ and $K_{2}$ are constants that do not depend on $h$.

We consider the following problem, referred to as problem $\mathrm{P}$ :

Given $f \in V_{h}^{\prime}$ and $g \in W_{h}^{\prime}$, find $(u, \psi) \in V_{h} \times W_{h}$ satisfying

$$
\begin{aligned}
a_{h}(u, v)+b_{h}(v, \psi) & =(f, v) \quad \forall v \in V_{h}, \\
b_{h}(u, \varphi) & =(g, \varphi) \quad \forall \varphi \in V_{h},
\end{aligned}
$$

where $(\cdot, \cdot)$ denotes the pairing between $V_{h}$ and its dual space $V_{h}^{\prime}$, or between $W_{h}$ and $W_{h}^{\prime}$.

We shall consider this problem for a subclass of data, i.e., for $(f, g) \in D$, where $D$ is a subclass of $V_{h}^{\prime} \times W_{h}^{\prime}$. We assume that $\mathrm{P}$ has a unique solution for all $(f, g) \in D$.

We are interested in the approximate solution of $P$. Toward this end we suppose we are given finite-dimensional spaces $V_{h} \subset V_{h}$ and $W_{h} \subset w_{h}, 0<h<1$, and consider the following problem. referred to as problem $\mathrm{P}_{h}$ :

Given $(f, g) \in D$, find $\left(u_{h}, \psi_{h}\right) \in V_{h} \times W_{h}$ satisfying

$$
\begin{aligned}
a_{h}\left(u_{h}, v\right)+b_{h}\left(v, \psi_{h}\right) & =(f, v) \quad \forall v \in V_{h}, \\
b_{h}\left(u_{h}, \varphi\right) & =(g, \varphi) \quad \forall \varphi \in W_{h} .
\end{aligned}
$$

We now regard $u_{h}$ as an approximation to $u$ and $\psi_{h}$ as an approximation to $\psi$.

Regarding problem $\mathrm{P}_{h}$, we suppose

$$
\sup _{v \in Z_{h}} \frac{\left|a_{h}(u, v)\right|}{\|v\|_{v_{h}}} \geqslant \gamma_{0}\|u\|_{v_{h}} \quad \forall u \in Z_{h} \text { and } \forall h,
$$

where $\gamma_{0}>0$ is independent of $h$, and $Z_{h} \equiv\left\{v \in V_{h}: b_{h}(v, \varphi)=0 \forall \varphi \in W_{h}\right\}$, and

$$
\sup _{v \in V_{h}} \frac{\left|b_{h}(v, \varphi)\right|}{\|v\|_{V_{h}}} \geqslant k_{0}\|\varphi\|_{\omega_{h}} \quad \forall \varphi \in W_{h} \text { and } \forall h,
$$

where $k_{0}>0$ is independent of $h$. We now state the fundamental estimate for the errors $u-u_{h}$ and $\psi-\psi_{h}$.

Theorem 1 (BREzzI [5]). Suppose (2.1), (2.2), (2.5), and (2.6) are satisfied. Then problem $\mathrm{P}_{h}$ has a unique solution $\left(u_{h}, \psi_{h}\right)$ for each $h$, and there is a constant $C$, independent of $h$, such that

$$
\left\|u-u_{h}\right\|_{v_{h}}+\left\|\psi-\psi_{h}\right\|_{w_{h}} \leqslant C\left(\inf _{\chi \in V_{h}}\|u-\chi\|_{v_{h}}+\inf _{\eta \in w_{h}}\|\psi-\eta\|_{w_{h}}\right) \quad \forall h .
$$

(2.5)-(2.6) is referred to as the stability condition for this approximation method.

In many applications of Theorem 1 the spaces $V_{h}$ and $W_{h}$ and the forms $a_{h}$ and $b_{h}$ do not depend on $h$, i.e., $V_{h}=V$ and $W_{h}=W$ are fixed Hilbert spaces and $a_{h}=a$ and $b_{h}=b$ are fixed bilinear forms and $V \times V$ and $V \times W$. The space $V_{h}$ and $W_{h}$, typically, are spaces of piecewise polynomials with respect to a triangulation $T_{h}$ of some domain by triangles of size less than or equal to $h$ and, of course, depend on $h$. 
In the applications in this paper, both the spaces $V_{h}, W_{h}$ and $V_{h}, W_{h}$ depend on $h$, i.e., are mesh dependent; the constants $K_{1}, K_{2}, \gamma_{0}$, and $k_{0}$, however, will be independent of $h$; cf. [2, Chapter 7]. In these applications the solution $(u, \psi)$ of $(2.3)$ is independent of $h$ and lies in $V_{h} \times W_{h}$ for all $h$. Thus the estimate (2.7) yields convergence estimates for $u-u_{h}$ and $\psi-\psi_{h}$, provided the families $\left\{V_{h}\right\}$ and $\left\{W_{h}\right\}$ satisfy an approximability assumption. For typical finite element applications this would involve the assumption that inf $\chi_{\chi \in V_{h}}\|u-\chi\|_{v_{h}}$ and $\inf _{\eta \in w_{h}}\|\psi-\eta\|_{w_{h}}$ tend to zero as $h$ tends to zero.

3. Mesh Dependent Norms and Spaces. In this section we describe the mesh dependent norms and spaces we shall use in the paper. Let $\Omega$ be a convex polygon in the plane. For $0<h<1$, we let $T_{h}$ be a triangulation of $\Omega$ by triangles $T$ of diameter less than or equal to $h$. We assume the family of triangulations $\left\{T_{h}\right\}$ satisfies the minimal angle condition, i.e., there is a constant $\sigma$ such that

$$
\max _{T \in T_{h}} \frac{h_{T}}{\rho_{T}} \leqslant \sigma \quad \forall h,
$$

where $h_{T}$ is the diameter of $T$ and $\rho_{T}$ is the diameter of the largest circle contained in $T$, and is quasi-uniform, i.e., there is a constant $\tau>0$ such that

$$
\frac{h}{h_{T}} \leqslant \tau \quad \forall T \in T_{h} \text { and } \forall h .
$$

Let $\Gamma_{h}=\bigcup_{T \in T_{h}} \partial T$. We define

$$
H_{h}^{2}=\left\{u \in H^{1}(\Omega):\left.u\right|_{T} \in H^{2}(T) \forall T \in T_{h}\right\}
$$

and on $H_{h}^{2}$ define the norm

$$
\|u\|_{2, h}^{2}=\sum_{T \in T_{h}}\|u\|_{2, T}^{2}+h^{-1} \int_{\Gamma_{h}}\left|J \frac{\partial u}{\partial \nu}\right|^{2} d s,
$$

where, if $T^{\prime}=\partial T^{1} \cap \partial T^{2}$ is an interior edge of the triangulation $T_{h}$, we set

$$
\left.J \frac{\partial u}{\partial \nu}\right|_{T^{\prime}}=\frac{\partial u}{\partial \nu^{1}}+\frac{\partial u}{\partial \nu^{2}},
$$

where $\nu^{j}$ is the unit normal to $T^{\prime}$ exterior to $T^{j}$, and if $T^{\prime}$ is a boundary edge of $T_{h}$, we set

$$
\left.J \frac{\partial u}{\partial v}\right|_{T^{\prime}}=\frac{\partial u}{\partial v}
$$

On $H^{1}(\Omega)$ we define

$$
\|u\|_{0, h}^{2}=\int_{\Omega}|u|^{2} d x+h \int_{\Gamma_{h}}|u|^{2} d s
$$

and then define $H_{h}^{0}$ to be the completion of $H^{1}(\Omega)$ with respect to $\|\cdot\|_{0, h}$. $H_{h}^{0}$ can be identified with $L_{2}(\Omega) \oplus L_{2}\left(\Gamma_{h}\right)$.

We note that norms similar to $\|\cdot\|_{0, h}$ and $\|\cdot\|_{2, h}$ have been used in a different manner in Douglas-Dupont [11] and Thomas [26]. 
For $k \geqslant 1$ a fixed integer, we define

$$
S_{h}=\left\{v \in C^{0}(\bar{\Omega}):\left.v\right|_{T} \in P_{k} \forall T \in T_{h}\right\}
$$

where $P_{k}$ is the space of polynomials of degree $k$ or less in the variable $x_{1}$ and $x_{2}$. It is clear that $S_{h}$ is contained in $H_{h}^{0}$ and $H_{h}^{2}$.

We now prove several lemmas that are fundamental to the analysis of this paper. These proofs are all closely related to the ideas used in the proof of the BrambleHilbert lemma [4]. Prior to stating the first of these lemmas, we describe the notation we will use and state some well-known results that will be used in the proofs.

Let $T$ be an arbitrary triangle and let $\hat{T}$ be the reference triangle with vertices $(0,0),(1,0)$, and $(0,1)$. Then there is an invertible affine mapping $F_{T}(\hat{x})=B_{T} \hat{x}+b_{T}$ $=F(\hat{x})=B \hat{x}+b$ such that $T=F_{T}(\hat{T})$. This mapping leads to the correspondence $\hat{x} \in \hat{T} \leftrightarrow x=F_{T}(\hat{x}) \in T$ between points in $\hat{T}$ and points in $T$ and the correspondence $(\hat{v}: \hat{T} \rightarrow R) \longleftrightarrow\left(v=\hat{v} \circ F_{T}^{-1}: T \longrightarrow R\right)$ between functions defined on $\hat{T}$ and functions defined on $T$. Note that $\hat{v}(\hat{x})=v(x)$.

It is easily seen that

$$
\left(\nabla_{x} v\right)(x)=\left(B^{-1}\right)^{t}\left(\nabla_{\hat{x}} \hat{v}\right)\left(F^{-1}(x)\right)
$$

If $\nu=\nu(x)$ denotes the outward unit normal to $\partial T$ at $x$ and $\hat{\nu}=\hat{\nu}(\hat{x})$ is the outward unit normal to $\partial \hat{T}$ and $\hat{x}$, then

$$
\nu(x)=\left(B^{-1}\right)^{t} \hat{\nu}(\hat{x})\left|B^{t} \nu(x)\right|
$$

where $t$ denotes transpose. Let the sides of $T$ be denoted by $T_{i}^{\prime}, i=1,2,3 .|T|$ denotes the area of $T$ and $\left|T_{i}^{\prime}\right|$ denotes the length of $T_{i}^{\prime}$. The seminorms $|v|_{l, T}$ and $|\hat{v}|_{l, \hat{T}}$ are related by

$$
|\hat{v}|_{l, \hat{T}} \leqslant|\operatorname{det} B|^{-1 / 2}\|B\|^{l}|v|_{l, T}
$$

and

$$
|v|_{l, T} \leqslant|\operatorname{det} B|^{1 / 2}\left\|B^{-1}\right\|^{l}|\hat{v}|_{l, \hat{T}},
$$

where $\|B\|$ is the norm of $B$ induced by the Euclidean vector norm; cf. [8, Theorem 3.1.2]. We will also use the estimates

$$
\|B\| \leqslant \frac{h_{T}}{\rho_{\hat{T}}}, \quad\left\|B^{-1}\right\| \leqslant \frac{h_{\hat{T}}}{\rho_{T}} ;
$$

cf. [8, Theorem 3.1.3]. We also note that $|\operatorname{det} B|=|T| /|\hat{T}|$. Finally we remark that there is a constant $C=C(\hat{T})$ such that

$$
\inf _{p \in P_{k}}\|\hat{u}+p\|_{k+1, \hat{T}} \leqslant C|\hat{u}|_{k+1, \hat{T}} \quad \forall \hat{u} \in H^{k+1}(\hat{T}) ;
$$

cf. [8, Theorem 3.1.1]

LEMmA 1. There is a constant $C$ such that

$$
\|u\|_{0, h} \leqslant C\|u\|_{0} \quad \forall u \in S_{h} .
$$


Proof. It is sufficient to show that

$$
h \int_{\Gamma_{h}}|u|^{2} d s \leqslant C\left\|_{u}\right\|_{0}^{2} \quad \forall u \in S_{h} .
$$

Now $\left(\int_{\hat{T}}|\hat{u}|^{2} d x\right)^{1 / 2}$ and $\left(\int_{\hat{T}}|\hat{u}|^{2} d x+\int_{\partial} \hat{T}|\hat{u}|^{2} d s\right)^{1 / 2}$ are both norms on the finitedimensional space $P_{k}(\hat{T})=\left\{\left.p\right|_{\hat{T}}: p \in P_{k}\right\}$ and hence there is a constant $C(\hat{T})$ such that

$$
\int_{\partial \hat{T}}|\hat{u}|^{2} d s \leqslant C(\hat{T}) \int_{\hat{T}}|\hat{u}| d \hat{x} \quad \forall \hat{u} \in P_{k}(\hat{T}) .
$$

Let $T \in T_{h}$ and suppose $T$ is the image of $\hat{T}$ under the mapping $F(\hat{x})=B \hat{x}+b$. Then, using (3.1), (3.2), and (3.6), we see that for any $u \in P_{k}$ we have

$$
\begin{aligned}
\int_{\partial \hat{T}}|u|^{2} d s & =\sum_{i=1}^{3} \int_{T_{i}^{\prime}}|u|^{2} d s \leqslant \sum_{i} \int_{\hat{T}_{i}^{\prime}}|\hat{u}|^{2}\left|T_{i}^{\prime}\right| d \hat{x} \\
& \leqslant C(\hat{T}) \max _{i}\left|T_{i}^{\prime}\right| \int_{\hat{T}}|\hat{u}|^{2} d \hat{x} \leqslant C(\hat{T}) \max _{i}\left|T_{i}^{\prime}\right||\operatorname{det} B|^{-1} \int_{T}|u|^{2} d x \\
& \leqslant C(\hat{T}) \max _{i}\left|T_{i}^{\prime}\right| \frac{|\hat{T}|}{|T|}\|u\|_{0, T}^{2} \leqslant \frac{C(\hat{T}) 4|\hat{T}|}{\pi}\left(\frac{h_{T}}{\rho_{T} 2}\right)\|u\|_{0, T}^{2} \\
& \leqslant \frac{C(\hat{T}) 4|\hat{T}|}{\pi}\left(\frac{h_{T}}{\rho_{T}}\right)^{2} \frac{1}{h_{T}}\|u\|_{0, T}^{2} \leqslant C(\hat{T}) \sigma h_{T}^{-1}\|u\|_{0, T}^{2} \\
& \leqslant C(\hat{T}) \sigma \tau h^{-1}\|u\|_{0, T}^{2} .
\end{aligned}
$$

Therefore

$$
\begin{aligned}
h \int_{\Gamma_{h}}|u|^{2} d s & \leqslant h \sum_{T \in T_{h}} \int_{\partial T}|u|^{2} d s \\
& \leqslant C(\hat{T}) \sigma \tau \sum_{T \in T_{h}}\|u\|_{0, T}^{2} \leqslant C(\hat{T}) \sigma \tau\|u\|_{0, \Omega}^{2}
\end{aligned}
$$

for all $u \in S_{h}$.

LEMMA 2. There is a constant $C$ such that

$$
\|u\|_{2, h} \leqslant C h^{-1}\|u\|_{1, \Omega} \quad \forall u \in P_{k} .
$$

Proof. Since $\left\{T_{h}\right\}$ is quasi-uniform, it is well known that

$$
\sum_{T \in T_{h}}\|u\|_{2, T}^{2} \leqslant C h^{-2}\|u\|_{1, \Omega}^{2} \quad \forall u \in P_{k} .
$$

Thus it is sufficient to show that

$$
h^{-1} \int_{\Gamma_{h}}\left|J \frac{\partial u}{\partial \nu}\right|^{2} d s \leqslant C h^{-2}\left\|_{u}\right\|_{1, \Omega}^{2} \quad \forall u \in P_{k}
$$

$\left(\int_{\hat{T}}|\hat{u}|^{2} d x+\int_{\partial \hat{T}}\left|\nabla_{\hat{x}} \hat{u}\right|^{2} d \hat{s}\right)^{1 / 2}$ and $\|\hat{u}\|_{1, \hat{T}}$ are both norms on the finite-dimensional space $P_{k}(\hat{T})$ and hence there is a constant $C(\hat{T})$ such that 


$$
E(\hat{u}) \equiv \int_{\partial}\left|\nabla_{\hat{x}} \hat{u}\right|^{2} d \hat{s} \leqslant C(\hat{T})\|\hat{u}\|_{1, \hat{T}}^{2} \quad \forall \hat{u} \in P_{k}(\hat{T})
$$

Clearly, $E(\hat{u}+p)=E(\hat{u}) \forall p \in P_{0}$. Thus

$$
E(\hat{u})=E(\hat{u}+p) \leqslant C(\hat{T})\|\hat{u}+p\|_{1, \hat{T}}^{2} \quad \forall p \in P_{0}
$$

and hence, using (3.9), we have

$$
E(\hat{u}) \leqslant C(\hat{T}) \inf _{p \in P_{0}}\|\hat{u}+p\|_{1, \hat{T}} \leqslant C(\hat{T})|\hat{u}|_{1, \hat{T}}
$$

Now let $T \in T_{h}$ and assume $T$ is the image of $\hat{T}$ under the mapping $F(x)=B x$ $+b$. Then, using (3.1), (3.2), (3.4), (3.6), and (3.8), we see that for any $u \in P_{k}$ we have

$$
\begin{aligned}
\int_{\partial T}\left|\frac{\partial}{\partial v}\right|^{2} d s & =\sum_{i} \int_{T_{i}^{\prime}}\left|\left[\left(\nabla_{x} u\right)(x)\right]^{t} \nu(x)\right|^{2} d s \\
& \leqslant \sum_{i} \int_{T_{i}^{\prime}}\left|\left(B^{-1}\right)^{t}\left(\nabla_{\hat{x}} \hat{u}\right)\left(F^{-1}(x)\right)\right|^{2} d s \\
& \leqslant\left|B^{-1}\right|^{2} \max _{i}\left|T_{i}^{\prime}\right| \int_{\partial \hat{T}}\left|\nabla_{\hat{x}} \hat{u}\right|^{2} d \hat{s} \leqslant C(\hat{T})\left\|B^{-1}\right\|^{2} \max \left|T_{i}^{\prime}\right||\hat{u}|_{1, \hat{T}}^{2} \\
& \leqslant C(\hat{T})\left\|B^{-1}\right\|^{2} \max \left|T_{i}^{\prime}\right||\operatorname{det} B|^{-1}\|B\|^{2}|u|_{1, T}^{2} \\
& \leqslant C(\hat{T})\left(\frac{h_{\hat{T}}}{\rho_{T}}\right)^{2}\left(\frac{h_{T}}{\rho_{\hat{T}}}\right)^{2} h_{T} \frac{|\hat{T}|}{|T|}|u|_{1, T}^{2} \\
& \leqslant C(\hat{T})\left(\frac{h_{\hat{T}}}{\rho_{\hat{T}}}\right)^{2}\left(\frac{h_{T}}{\rho_{T}}\right)^{4} \frac{4|\hat{T}|}{\pi} \frac{1}{h_{T}}|u|_{1, T}^{2} \\
& \leqslant C(\hat{T})\left(\frac{h_{\hat{T}}}{\rho_{\hat{T}}}\right)^{2} \sigma^{4} \frac{4|\hat{T}|}{\pi} h^{-1}|u|_{1, T}^{2}
\end{aligned}
$$

Therefore, we obtain

$$
\begin{aligned}
h^{-1} \int_{\Gamma_{h}}\left|J \frac{\partial u}{\partial \nu}\right|^{2} d s & \leqslant \sum_{T \in T_{h}} h^{-1} \int_{\partial T}\left|\frac{\partial u}{\partial \nu}\right|^{2} d s \\
& \leqslant C(\hat{T}) \sigma^{4} \tau \sum_{T \in T_{h}} h^{-2}|u|_{1, T}^{2} \leqslant C(\hat{T}) \sigma^{4} \tau h^{-2}|u|_{1, \Omega}^{2}
\end{aligned}
$$

This completes the proof.

LEMMA 3. There is a constant $C$ such that

$$
\inf _{\chi \in S_{h}}\|u-\chi\|_{0, h} \leqslant C h^{l}|u|_{l, \Omega}
$$

for all $u \in H^{r}(\Omega)$ and all $h$, where $1 \leqslant r$ and $1 \leqslant l \leqslant \min (r, k+1)$.

Proof. We define two interpolation operators that will be used in the proof. For $u \in H^{2}(T)$, let $I_{T} u \in P_{k}$ be defined by 
and

$$
\begin{array}{ll}
\int_{T}\left(u-I_{T} u\right) f d x=0 & \forall f \in P_{k-3}, \\
\int_{T^{\prime}}\left(u-I_{T^{u}} u\right) f d x=0 & \forall f \in P_{k-2} \text { and } \forall \text { sides } T^{\prime} \text { of } T,
\end{array}
$$

$$
u(a)-\left(I_{T} u\right)(a)=0 \quad \forall \text { vertices } a \text { of } T \text {. }
$$

Then, for $u \in H^{2}(\Omega)$, we let $I_{h} u \in S_{h}$ be defined by

$$
\left.\left(I_{h} u\right)\right|_{T}=I_{T}\left(\left.u\right|_{T}\right) \text {. }
$$

For $u \in H^{1}(\Omega)$, we define the interpolant in a different manner. Here we consider only the case $k=1$. Let the vertices of $T_{h}$ be denoted by $z_{1}, \ldots, z_{m}$ and let $w_{1}, \ldots, w_{m}$ be the basis for $S_{h}$ defined by $w_{i}\left(z_{j}\right)=\delta_{i j}$. Set $S_{j}=\left(\operatorname{supp} w_{j}\right) \cap \Omega$ and let $\left|S_{j}\right|$ be the area of $S_{j}$. Now, following Clément [10], we define $\widetilde{I}_{h} u$ by

$$
\tilde{I}_{h} u=\sum_{j=1}^{m} \frac{\int_{s_{j}} u d x}{\left|S_{j}\right|} w_{j} .
$$

We first consider the case $r \geqslant 2$ and $l \geqslant 2$. In this case we obtain the desired result by estimating $\left\|u-I_{h} u\right\|_{0, h}$. By the standard approximability results for $S_{h}$, we have

$$
\int_{\Omega}\left|u-I_{h} u\right|^{2} d x \leqslant C h^{2 l}|u|_{l, \Omega}^{2}
$$

Thus it is sufficient to show that

$$
\int_{\Gamma_{h}}\left|u-I_{h} u\right|^{2} d s \leqslant C h^{2 l-1}|u|_{l, \Omega}^{2} .
$$

Suppose $u \in H^{l}(\hat{T})$ and set $E(\hat{u})=\int_{\partial T}\left|\hat{u}-I_{\hat{T}} \hat{u}\right|^{2} d \hat{s}$. By the trace theorem and the Sobolev imbedding theorem we have

$$
E(\hat{u}) \leqslant C(\hat{T})\|\hat{u}\|_{l, \hat{T}}^{2},
$$

and since $E(\hat{u}+p)=E(\hat{u}) \forall p \in P_{l-1}$, we thus have

$$
E(\hat{u}) \leqslant C(\hat{T}) \inf _{p \in P_{l-1}}\|\hat{u}+p\|_{l, \hat{T}}^{2} \leqslant C(\hat{T})|u|_{l, \hat{T}}^{2} .
$$

Now let $T \in T_{h}$ be the image of $\hat{T}$ under the mapping $F(\hat{x})=B \hat{x}+b$. Then

$$
\begin{aligned}
\int_{\partial T}\left|u-I_{T} u\right|^{2} d s & \leqslant \sum_{i} \int_{\hat{T}_{i}^{\prime}}\left|\hat{u}-I_{\hat{T}} \hat{u}\right|^{2}\left|T_{i}^{\prime}\right| d \hat{s} \\
& \leqslant \max _{i}\left|T_{i}^{\prime}\right| \int_{\partial \hat{T}}\left|\hat{u}-I_{\hat{T}} \hat{u}\right|^{2} d \hat{s} \leqslant \max _{i}\left|T_{i}^{\prime}\right| C(\hat{T})|\hat{u}|_{l, \hat{T}}^{2} \\
& \leqslant\left.\max _{i}\left|T_{i}^{\prime}\right| C(\hat{T})|\operatorname{det} B|^{-1}\left|B \|^{2 l}\right| u\right|_{l, T} ^{2} \\
& \leqslant C(\hat{T})|\hat{T}| \frac{4 h_{T}}{\pi \rho_{T}^{2}}\left(\frac{h_{T}}{\rho_{\hat{T}}}\right)^{2 l}|u|_{l, T}^{2} \\
& \leqslant \frac{C(\hat{T})|\hat{T}| 4 \sigma^{2}}{\pi \rho_{\hat{T}}^{2 l}} h_{T}^{2 l-1}|u|_{l, T}^{2} .
\end{aligned}
$$


Therefore

$$
\begin{aligned}
\int_{\Gamma_{h}}\left|u-I_{h} u\right|^{2} d s & \leqslant \sum_{T \in T_{h}} \int_{\partial T}\left|u-I_{T} u\right|^{2} d s \\
& \leqslant C(\hat{T}) \sigma^{2} h^{2 l-1} \sum_{T \in T_{h}}|u|_{l, T}^{2}=C(\hat{T}) \sigma^{2} h^{2 l-1}|u|_{l, \Omega}^{2} .
\end{aligned}
$$

This completes the proof for the case $r, l \geqslant 2$.

For the case $r \geqslant 2$ and $l=1$ or $r=l=1$, we estimate $\left\|u-\tilde{I}_{h} u\right\|_{0, h}$. Clément [10] has shown that

$$
\left\|u-\tilde{I}_{h} u\right\|_{0} \leqslant C h|u|_{1} .
$$

By a slight modification of the proof in [9], we obtain

$$
\left(h \int_{\Gamma_{h}}\left|u-\hat{I}_{h} u\right|^{2} d s\right)^{1 / 2} \leqslant C h|u|_{1} .
$$

The desired result now follows.

Lemma 4. There is a constant $C$ such that

$$
\inf _{\chi \in S_{h} \cap H_{0}^{1}}\|u-\chi\|_{2, h} \leqslant C h^{l-2}|u|_{l, \Omega}
$$

for all $u \in H^{r}(\Omega) \cap H_{0}^{1}(\Omega)$ and all $h$, where $2 \leqslant r$ and $2 \leqslant l \leqslant \min (r, k+1)$.

Proof. Let $I_{h}$ be defined as in the proof of Lemma 3. Note that $I_{h} u \in S_{h} \cap$ $H_{0}^{1}$ if $u \in H^{r} \cap H_{0}^{1}$. Since, by standard approximability results, we have

$$
\sum_{T}\left\|u-I_{h} u\right\|_{2, T}^{2} \leqslant C h^{2 l-4}|u|_{l, \Omega}^{2}
$$

it is sufficient to show that

$$
\int_{\Gamma_{h}}\left|J \frac{\partial\left(u-I_{h} u\right)}{\partial \nu}\right|^{2} d s \leqslant C h^{2 l-3}|u|_{l, \Omega}^{2} .
$$

We next observe that

$$
\int_{\partial \hat{T}}\left|\nabla_{\hat{x}}\left(u-I_{\hat{T}} \hat{u}\right)\right|^{2} d s \leqslant C(\hat{T})|\hat{u}|_{l, T}^{2} \quad \forall \hat{u} \in H^{l}(\hat{T}) .
$$

Now let $T \in T_{h}$ be the image of $\hat{T}$ under the mapping $F(\hat{x})=B \hat{x}+b$. Then

$$
\begin{aligned}
\int_{\partial T} \mid \frac{\partial}{\partial \nu}( & \left.u-I_{T^{u}}\right)\left.\right|^{2} d s=\sum_{i} \int_{T_{i}^{\prime}} \mid\left[\left.\nabla_{x}\left(u-I_{T^{u}} u\right){ }^{t} \nu(x)\right|^{2} d s\right. \\
& =\sum_{i} \int_{T_{i}^{\prime}}\left|\left(B^{-1}\right)^{t} \nabla_{\hat{x}}\left(\hat{u}-I_{\hat{T}} \hat{u}\right)\right|^{2} d s \\
& \leqslant \sum_{i} \int_{T_{i}^{\prime}}\left|\left(B^{-1}\right)^{t} \nabla_{\hat{x}}\left(\hat{u}-I_{\hat{T}} \hat{u}\right)(\hat{x})\right|^{2}\left|T_{i}^{\prime}\right| d \hat{s} \\
& \leqslant\left|B^{-1}\right|^{2} \max _{i}\left|T_{i}^{\prime}\right| \int_{\partial \hat{T}}\left|\nabla_{\hat{x}}\left(\hat{u}-I_{\hat{T}} \hat{u}\right)\right|^{2} d \hat{s} \leqslant C(\hat{T})\left\|B^{-1}\right\|^{2} \max \left|T_{i}^{\prime}\right||\hat{u}|_{l, \hat{T}}^{2} \\
& \leqslant C(\hat{T})\left(\frac{h_{\hat{T}}}{\rho_{T}}\right)^{2}\left(\frac{h_{T}}{\rho_{\hat{T}}}\right)^{2 l} \frac{|\hat{T}|}{|T|}|u|_{l, T}^{2} h_{T} \leqslant \frac{C(\hat{T})|\hat{T}| 4 h_{\hat{T}}^{2}}{\pi \rho_{\hat{T}}^{2 l}} \sigma^{4} h_{T}^{2 l-3}|u|_{l, T}^{2} .
\end{aligned}
$$


Therefore

$$
\begin{aligned}
\int_{\Gamma_{h}}\left|J \frac{\partial\left(u-u_{T}\right)}{\partial \nu}\right|^{2} d s & \leqslant \sum_{T \in T} \int_{\partial T}\left|\frac{\partial}{\partial \nu}\left(u-I_{T} u\right)\right|^{2} d s \\
& \leqslant C(\hat{T}) \sigma^{4} h^{2 l-3}|u|_{l, \Omega}^{2}
\end{aligned}
$$

which completes the proof.

4. Applications. In this section we analyze three mixed methods.

(a) Ciarlet-Raviart Method. Consider the biharmonic problem

$$
\left\{\begin{array}{l}
\Delta^{2} \psi=g \text { in } \Omega \\
\psi=\partial \psi / \partial \nu=0 \quad \text { on } \Gamma=\partial \Omega
\end{array}\right.
$$

where $\Omega$ is a convex polygon in the plane and $g$ is a given function. If $g \in H^{-2}(\Omega)$, then there is a unique solution $\psi \in H_{0}^{2}(\Omega)$ of (4.1). In addition, the following regularity result is known for this problem: If $g \in H^{-1}(\Omega)$, then $\psi \in H^{3}(\Omega) \cap H_{0}^{2}(\Omega)$ and there is a constant $C$ such that

$$
\|\psi\|_{3} \leqslant C\|g\|_{-1} \quad \forall g \in H^{-1}(\Omega) .
$$

Using the well-known correspondence between the biharmonic problem and the Stokes problem, this regularity result can be deduced from the regularity result for the Stokes problem proved in [18]. We assume $g \in H^{-1}(\Omega)$ throughout this section.

We now seek an approximation to the solution $\psi$ of (4.1) by a mixed method, i.e., we introduce an auxiliary variable, $(u \equiv-\Delta \psi$ for the method of this subsection), write (4.1) as a second-order system, cast this sytem into variational form, and then consider the Ritz-Galerkin method corresponding to this variational formulation.

Thus we let $u \equiv-\Delta \psi$ and write (4.1) as

$$
\left\{\begin{array}{l}
\Delta u=-g, \\
\Delta \psi+u=0 \quad \text { in } \Omega, \\
\psi=\partial \psi / \partial \nu=0 \text { on } \Gamma .
\end{array}\right.
$$

The desired variational formulation of (4.3) is obtained by multiplying the 1 st equation in (4.3) by $\varphi \in H_{h}^{2} \cap H_{0}^{1}$, the 2 nd equation by $v \in H_{h}^{0}$, integrating the resulting equations over $\Omega$, and integrating the first one by parts over each $T \in T_{h}$. By means of this process we arrive at the following problem:

Given $g \in H^{-1}(\Omega)$, find $(u, \psi) \in H_{h}^{0} \times\left(H_{h}^{2} \cap H_{0}^{1}\right)$ satisfying

$$
\begin{cases}\int_{\Omega} u v d x-\sum_{T \in T_{h}} \int_{T} v \Delta \psi d x-\int_{\Gamma_{h}} v\left(J \frac{\partial \psi}{\partial \nu}\right) d s=0 & \forall v \in H_{h}^{0} \\ \sum_{T \in T_{h}} \int_{T} u \Delta \varphi d x-\int_{\Gamma_{h}} u\left(J \frac{\partial \varphi}{\partial \nu}\right) d s=-\int_{\Omega} g \varphi d x \quad \forall \varphi \in H_{h}^{2} \cap H_{0}^{1}\end{cases}
$$


Using the regularity result (4.2), one can easily show that if $\psi$ is the solution of (4.1) and $u \equiv-\Delta \psi$, then $(u, \psi)$ is a solution of (4.4), and if $(u, \psi)$ is a solution of (4.4), then $\psi$ is the solution of (4.1) and $u=-\Delta \psi$. (4.4) is an example of problem $\mathrm{P}$ in Section 2 with $V_{h}=H_{h}^{0},\|\cdot\|_{v_{h}}=\|\cdot\|_{0, h}, w_{h}=H_{h}^{2} \cap H_{0}^{1},\|\cdot\|_{w_{h}}=\|\cdot\|_{2, h}, a_{h}(u, v)$ $=\int_{\Omega} u v d x$, and

$$
b_{h}(u, \varphi)=\sum_{T \in T_{h}} \int_{T} u \Delta \varphi d x-\int_{\Gamma_{h}} u\left(J \frac{\partial \varphi}{\partial \nu}\right) d s
$$

(and with $g$ replaced by $-g$ ). Here the subclass of data for which (4.4) is uniquely solvable is $D=0 \times H^{-1}(\Omega)$.

As pointed out above, $H_{h}^{0}$ can be identified with $L_{2}(\Omega) \oplus L_{2}\left(\Gamma_{h}\right)$. Under this identification, $H^{1}(\Omega)$ is considered a linear manifold in $H_{h}^{0}$ through the mapping

$$
H^{1}(\Omega) \ni u \rightarrow\left(u,\left.u\right|_{\Gamma_{h}}\right) \in L_{2}(\Omega) \oplus L_{2}\left(\Gamma_{h}\right)=H_{h}^{0}
$$

Thus an element $u=(\tilde{u}, \underset{\tilde{u}}{\widetilde{*}}) \in L_{2}(\Omega) \oplus L_{2}\left(\Gamma_{h}\right)$ is considered to be in $H^{1}(\Omega)$ if $\tilde{u} \in$ $H^{1}(\Omega)$ and $\left.u\right|_{\Gamma_{h}}=\widetilde{u}$. To be completely precise, $b_{h}$ should be defined by

$$
b_{h}(u, \varphi)=\sum_{T \in T_{h}} \int_{T} \tilde{u} \Delta \varphi d x-\int_{\Gamma_{h}} \tilde{u}\left(J \frac{\partial \varphi}{\partial \nu}\right) d s
$$

for $u=(\tilde{u}, \widetilde{u}) \in H_{h}^{0}=L_{2}(\Omega) \oplus L_{2}\left(\Gamma_{h}\right)$ and $\varphi \in H_{h}^{2}$. Note that

$$
b_{h}(u, \varphi)=-\int_{\Omega} \nabla u \cdot \nabla \varphi d x
$$

for $u \in H^{1}(\Omega)$ and $\varphi \in H_{h}^{2}$. We further note that it is immediate that (2.1) and (2.2) are satisfied with constants that do not depend on $h$.

For finite-dimensional spaces we choose $V_{h}=S_{h}$ and $W_{h}=S_{h} \cap H_{0}^{1}(\Omega)$, where $S_{h}$ is defined in (3.3). Problem $\mathrm{P}_{h}$ thus has the form:

Given $g \in H^{-1}(\Omega)$, find $\left(u_{h}, \psi_{h}\right) \in V_{h} \times W_{h}$ satisfying

$$
\left\{\begin{array}{l}
\int_{\Omega} u_{h} v d x+\sum_{T \in T_{h}} \int_{T} v \Delta \psi_{h} d x-\int_{\Gamma_{h}} v\left(J \frac{\partial \psi_{h}}{\partial \nu}\right) d x=0 \quad \forall v \in V_{h}, \\
\sum_{T \in T_{h}} \int_{T} u_{h} \Delta \varphi d x-\int_{\Gamma_{h}} u_{h}\left(J \frac{\partial \varphi}{\partial \nu}\right) d s=-\int_{\Omega} g \varphi d x \quad \forall \varphi \in W_{h} .
\end{array}\right.
$$

Using (4.5), one easily sees that the approximation procedure determined by (4.6) is the same as that considered by Glowinski [14] and Mercier [19] and further developed by Ciarlet-Raviart [9]. Note that this method yields direct approximations to $\psi$ and to $u=-\Delta \psi$ (the stream function and vorticity in hydrodynamical problems).

We have already observed that (2.1) and (2.2) are satisfied. In order to apply Theorem 1, we must check the stability condition (2.5)-(2.6). 
THEOREM 2. There is a constant $\gamma_{0}>0$, independent of $h$, such that

$$
\sup _{v \in Z_{h}} \frac{\left|\int_{\Omega} u v d x\right|}{\left\|_{v}\right\|_{0, h}} \geqslant \gamma_{0}\|u\|_{0, h} \quad \forall u \in Z_{h}
$$

i.e., (2.5) is satisfied.

Proof. Using Lemma 1, we have

$$
\begin{aligned}
\sup _{v \in Z_{h}} \frac{\left|\int_{\Omega} u v d x\right|}{\|v\|_{0, h}} & \geqslant \sup _{v \in Z_{h}} \frac{\left|\int_{\Omega} u v d x\right|}{C\left\|_{v}\right\|_{0}} \\
& =C^{-1}\|u\|_{0} \geqslant C^{-2}\|u\|_{0, h} \quad \forall u \in Z_{h},
\end{aligned}
$$

where $C$ is independent of $h$. Thus (2.5) holds with $\gamma_{0}=C^{-2}$.

Now we consider (2.6). Let $\widetilde{S}_{h} \equiv\left\{v \in S_{h}: \int_{\Omega} v d x=0\right\}$.

Lemma 5. There is a constant $C_{1}>0$, independent of $h$, such that

$$
\inf _{\varphi \in \tilde{S}_{h}} \sup _{v \in \widetilde{S}_{h}} \frac{\left|\int_{\Omega} \nabla v \cdot \nabla \varphi d x\right|}{\|v\|_{0, h}\|\varphi\|_{2, h}} \geqslant C_{1} \quad \forall h .
$$

Proof. We first note that

$$
\inf _{\varphi \in \tilde{S}_{h}} \sup _{v \in \widetilde{S}_{h}} \frac{\left|\int_{\Omega} \nabla v \cdot \nabla \varphi d x\right|}{\|v\|_{0, h}\|\varphi\|_{2, h}}=\inf _{v \in \tilde{S}_{h}} \sup _{\varphi \in \tilde{S}_{h}} \frac{\left|\int_{\Omega} \nabla v \cdot \nabla \varphi d x\right|}{\|v\|_{0, h}\|\varphi\|_{2, h}} .
$$

This is a consequence of the fact that an operator and its adjoint have equal norms.

Given $v \in \widetilde{S}_{h}$, we choose $\varphi$ to satisfy

$$
\left\{\begin{array}{l}
\varphi \in \widetilde{S}_{h}, \\
\int_{\Omega} \nabla \varphi \cdot \nabla \xi d x=\int_{\Omega} v \xi d x \quad \forall \xi \in \widetilde{S}_{h} .
\end{array}\right.
$$

Letting $\xi=v$ and using Lemma 1 , we obtain

$$
\int_{\Omega} \nabla v \cdot \nabla \varphi d x=\int_{\Omega} v^{2} d x \geqslant C_{2}\|v\|_{0, h}^{2},
$$

where $C_{2}>0$ is independent of $h$.

Now let $\bar{\varphi}$ be defined by

$$
\left\{\begin{array}{l}
\bar{\varphi} \in \widetilde{H}^{1}(\Omega) \equiv\left\{u \in H^{1}(\Omega): \int_{\Omega} u d x=0\right\} \\
\int_{\Omega} \nabla \bar{\varphi} \cdot \nabla \xi d x=\int_{\Omega} v \xi d x \quad \forall \xi \in \widetilde{H}^{1}(\Omega) .
\end{array}\right.
$$

Then $\partial \bar{\varphi} / \partial \nu=0$ on $\Gamma$ and, since $\Omega$ is convex,

$$
\|\bar{\varphi}\|_{2} \leqslant C\left\|_{u}\right\|_{0} .
$$


$\varphi$ is the Neumann projection of $\bar{\varphi}$ into $\widetilde{S}_{h}$ and it is well known that

$$
\|\varphi-\bar{\varphi}\|_{1} \leqslant C h\|\bar{\varphi}\|_{2} \text {. }
$$

Let $\overline{\bar{\varphi}}$ be the piecewise linear interpolant of $\bar{\varphi}$.

Since $\bar{\varphi} \in H^{2}(\Omega)$ and $\partial \bar{\varphi} / \partial \nu=0$ on $\Gamma$, we see from the definition of $\|\cdot\|_{2, h}$ and from (4.9) that

$$
\|\bar{\varphi}\|_{2, h}=\|\bar{\varphi}\|_{2} \leqslant C\left\|_{v}\right\|_{0} .
$$

From Lemma 4, with $k=1$ and $r=2$, and (4.10), we have

$$
\|\bar{\varphi}-\overline{\bar{\varphi}}\|_{2, h} \leqslant C\|\bar{\varphi}\|_{2} \leqslant C\left\|_{v}\right\|_{0} \text {. }
$$

Using Lemma 2, (4.9), (4.10), and standard approximability results, we find that

$$
\begin{aligned}
\|\varphi-\overline{\bar{\varphi}}\|_{2, h} \leqslant C h^{-1}\|\varphi-\overline{\bar{\varphi}}\|_{1} & \leqslant C h^{-1}\left(\|\varphi-\bar{\varphi}\|_{1}+\|\bar{\varphi}-\overline{\bar{\varphi}}\|_{1}\right) \\
& \leqslant C h^{-1}\left(h\|\bar{\varphi}\|_{2}+h\|\bar{\varphi}\|_{2}\right) \leqslant C\left\|_{v}\right\|_{0} .
\end{aligned}
$$

Now, using (4.11)-(4.13), we have

$$
\|\varphi\|_{2, h} \leqslant\left\|\varphi-\overline{\bar{\varphi}}_{2, h}+\right\| \overline{\bar{\varphi}}-\bar{\varphi}\left\|_{2, h}+\right\| \bar{\varphi}\left\|_{2, h} \leqslant C_{3}\right\| v\left\|_{0} \leqslant C_{3}\right\| v \|_{0, h},
$$

where $C_{3}$ is independent of $h$.

Combining (4.8) and (4.14), we get

$$
\inf _{v \in \widetilde{S}_{h}} \sup _{\varphi \in \widetilde{S}_{h}} \frac{\left|\int_{\Omega} \nabla v \cdot \nabla \varphi d x\right|}{\|v\|_{0, h}\|\varphi\|_{2, h}} \geqslant \frac{C_{2}}{C_{3}} \equiv C_{1}>0 .
$$

The desired result now follows from (4.7) and (4.15).

THEOREM 3. There is a constant $k_{0}>0$, independent of $h$, such that

$$
\sup _{v \in V_{h}} \frac{\left|b_{h}(v, \varphi)\right|}{\|v\|_{0, h}} \geqslant k_{0}\|\varphi\|_{2, h} \quad \forall \varphi \in W_{h} \text { and } \forall h,
$$

i.e., (2.6) is satisfied.

Proof. Let $\varphi \in W_{h}$ and set $e=(1 /|\Omega|) \int_{\Omega} \varphi d x$. Then $|e| \leqslant C\|\varphi\|_{0}$ and $\tilde{\varphi} \equiv$ $\varphi-e \in \widetilde{S}_{h}$. By Lemma 5 there is a $v_{1} \in \widetilde{S}_{h}$ such that

$$
b_{h}\left(v_{1}, \varphi\right)=b_{h}\left(v_{1}, \tilde{\varphi}\right)=-\int_{\Omega} \nabla v_{1} \cdot \nabla \tilde{\varphi} d x \geqslant\|\tilde{\varphi}\|_{2, h}^{2} \geqslant\|\varphi\|_{2, h}^{2}-C_{4}\|\varphi\|_{0}^{2}
$$

and

$$
\left\|v_{1}\right\|_{0, h} \leqslant C\|\tilde{\varphi}\|_{2, h} \leqslant C_{5}\|\varphi\|_{2, h}
$$

We also know that

$$
-b_{h}(\varphi, \varphi)=\int_{\Omega}|\nabla \varphi|^{2} d x \geqslant C_{6}\|\varphi\|_{0}^{2}
$$

and

$$
\|\varphi\|_{0, h} \leqslant C_{7}\|\varphi\|_{2, h}
$$


Now let $v=v_{1}-C_{4} C_{6}^{-1} \varphi$. Then, using (4.17)-(4.20), we have

$$
b_{h}(v, \varphi) \geqslant\|\varphi\|_{2, h}^{2}
$$

and

$$
\|v\|_{0, h} \leqslant\left(C_{5}+C_{4} C_{7} C_{6}^{-1}\right)\|\varphi\|_{2, h} .
$$

Combining (4.21) and (4.22), we have (4.16) with $C=\left(C_{5}+C_{4} C_{7} C_{6}^{-1}\right)^{-1}$.

We are now ready to apply Theorem 1 to analyze the Ciarlet-Raviart method.

We obtain

$$
\left\|u-u_{h}\right\|_{0, h}+\left\|\psi-\psi_{h}\right\|_{2, h} \leqslant C\left(\inf _{x \in V_{h}}\|u-\chi\|_{0, h}+\inf _{\eta \in W_{h}}\|\psi-\eta\|_{2, h}\right) .
$$

Suppose $\psi \in H^{r}(\Omega), r \geqslant 3$, and suppose $k \geqslant 2$. Using Lemmas 3 and 4 , we obtain

$$
\left\|u-u_{h}\right\|_{0, h}+\left\|\psi-\psi_{h}\right\|_{2, h} \leqslant C h^{s-2}\|\psi\|_{s},
$$

where $s=\min (r, k+1)$. From (4.23) we get

$$
\left\|u-u_{h}\right\|_{0} \leqslant C h^{s-2}\|\psi\|_{s} \text {. }
$$

In addition, (4.23) yields the estimates

$$
\begin{aligned}
& \left(\int_{\Gamma_{h}}\left|u-u_{h}\right|^{2} d s\right)^{1 / 2} \leqslant C h^{s-5 / 2}\|\psi\|_{s}, \\
& \left(\sum_{T \in T_{h}}\left\|\psi-\psi_{h}\right\|_{2, T}^{2}\right)^{1 / 2} \leqslant C h^{s-2}\|\psi\|_{s},
\end{aligned}
$$

and

$$
\left(\int_{\Gamma_{h}}\left|J \frac{\partial \psi_{h}}{\partial \nu}\right|^{2} d s\right)^{1 / 2} \leqslant C h^{s-3 / 2}\|\psi\|_{s}
$$

We now derive an estimate for $\left\|\psi-\psi_{h}\right\|_{1}$ by means of the well-known duality argument. Given $d \in H^{-1}(\Omega)$, let $\theta$ be the solution of

$$
\begin{cases}\Delta^{2} \theta=d & \text { in } \Omega, \\ \theta=\partial \theta / \partial \nu=0 & \text { on } \Gamma .\end{cases}
$$

If we let $w=-\Delta \theta$, then from (4.2) we have

$$
\|\theta\|_{3}+\|w\|_{1} \leqslant C\|d\|_{-1} .
$$

Also, from the discussion following Eqs. (4.4), we know that the pair $(w, \theta)$ satisfies

$$
(d, \varphi)_{0}=-a_{h}(v, w)-b_{h}(w, \varphi)-b_{h}(v, \theta) \quad \forall(v, \varphi) \in H_{h}^{0} \times\left(H_{h}^{2} \cap H_{0}^{1}\right) .
$$

Setting $v=u-u_{h}$ and $\varphi=\psi-\psi_{h}$, using the exact equations (4.4) and the Ritz-

Galerkin equations (4.6), we get

$$
\begin{array}{r}
\left(d, \psi-\psi_{h}\right)_{0}=-a_{h}\left(u-u_{h}, w\right)-b_{h}\left(w, \psi-\psi_{h}\right)-b_{h}\left(u-u_{h}, \theta\right) \\
=-a_{h}\left(u-u_{h}, w-z\right)-b_{h}\left(w-z, \psi-\psi_{h}\right)-b_{h}\left(u-u_{h}, \theta-\mu\right) \\
\forall(z, \mu) \in V_{h} \times W_{h} .
\end{array}
$$


Thus, using (2.1), (2.2), (4.26), and Lemmas 3 and 4, we get

$$
\begin{aligned}
\left|\left(d, \psi-\psi_{h}\right)\right| \leqslant & C\left(\left\|u-u_{h}\right\|_{0, h}+\left\|\psi-\psi_{h}\right\|_{2, h}\right) \inf _{z \in V_{h}}\|w-z\|_{0, h} \\
& +\left\|u-u_{h}\right\|_{0, h} \inf _{\mu \in w_{h}}\|\theta-\mu\|_{2, h} \\
\leqslant & C h\|d\|_{-1}\left(\left\|u-u_{h}\right\|_{0, h}+\left\|\psi-\psi_{h}\right\|_{2, h}\right) .
\end{aligned}
$$

Finally, combining (4.23) and (4.27), we have

$$
\left\|\psi-\psi_{h}\right\|_{1}=\sup _{d \in H^{-1}(\Omega)} \frac{\left|\left(d, \psi-\psi_{h}\right)\right|}{\|d\|_{-1}} \leqslant C h^{s-1}\|\psi\|_{s},
$$

where $s=\min (r, k+1)$.

Estimates (4.24) and (4.28) improve on those in Ciarlet-Raviart [9]. Scholz [23] obtained (4.24) under the assumption that $\Gamma$ is smooth. (4.24) and (4.28) were also obtained by Falk-Osborn [12]. Note that the approach of this paper does not yield error estimates for the case $k=1$ for the method studied in this subsection (and also for the method of Subsection (b)); for this case the reader is referred to Scholz [24]. Using $L_{\infty}$-estimate techniques, Scholz [24] has shown that $\left\|u-u_{h}\right\|_{0}=O\left(h^{s-3 / 2}\right)$ under different assumptions than those made in (4.24). In [25] it is shown that in any subdomain $\Omega_{0} \subset \subset \Omega,\left\|u-u_{h}\right\|_{0, \Omega_{0}}$ is of "nearly" the same order as $\left\|\psi-\psi_{h}\right\|_{0, \Omega}$, provided $\psi$ is sufficiently smooth. Finally we note that our approach allows the treatment of the case when $g \in\left(H_{h}^{2}\right)^{\prime}-H^{-1}(\Omega)$. For example, we could treat the case where $g$ is the Dirac function, which corresponds to a concentrated load in plate theory.

Estimates (4.25) are new for this problem. (4.25c) provides an estimate on the rate at which the jumps in the normal derivatives of $\psi_{h}$ across interelement boundaries is converging to zero and also contains the estimate

$$
\int_{\Gamma}\left|\frac{\partial \psi_{h}}{\partial \nu}\right|^{2} d s \leqslant C h^{s-3 / 2}\|\psi\|_{s}
$$

(b) Herrmann-Miyoshi Method. In this subsection we consider another mixed method for the approximate solution of (4.1). In this method the auxiliary variable is the matrix of second-order partial derivatives of $\psi$.

For $T \in T_{h}$ and $\mathbf{v}=\left(v_{i j}\right), 1 \leqslant i, j \leqslant 2$, with $v_{i j} \in H^{1}(T)$ and $v_{12}=v_{21}$, we set

$$
M_{\nu}(\mathbf{v})=\sum_{i, j=1}^{2} v_{i j} \nu_{j} \nu_{i}
$$

and

$$
M_{\nu \tau}(\mathbf{v})=\sum_{i, j=1}^{2} v_{i j} \nu_{j} \tau_{i}
$$

where $\nu=\left(\nu_{1}, \nu_{2}\right)$ is the unit outward normal and $\tau=\left(\tau_{1}, \tau_{2}\right)=\left(\nu_{2},-\nu_{1}\right)$ is the unit tangent along $\partial T$. We note that

$$
\sum_{i, j=1}^{2} \int_{T}\left(v_{i j} \frac{\partial^{2} \varphi}{\partial x_{i} \partial x_{j}}+\frac{\partial v_{i j}}{\partial x_{j}} \frac{\partial \varphi}{\partial x_{i}}\right) d x=\int_{\partial T}\left(M_{\nu}(\mathbf{v}) \frac{\partial \varphi}{\partial \nu}+M_{\nu \tau}(\mathbf{v}) \frac{\partial \varphi}{\partial \tau}\right) d s
$$


for all $\varphi \in H^{2}(T)$. On

$$
\grave{V}_{h}(\Omega) \equiv\left\{\mathbf{v}=\left(v_{i j}\right), 1 \leqslant i, j \leqslant 2: v_{12}=v_{21}, v_{i j} \in H^{1}(T) \forall T \in T_{h}\right. \text {, and }
$$

$M_{\nu}(\mathbf{v})$ is continuous across interelement boundaries

we define

$$
\|\mathrm{v}\|_{0, h}^{2}=\sum_{i, j} \int_{\Omega}\left|v_{i j}\right|^{2} d x+h \int_{\Gamma_{h}}|M(\mathrm{v})|^{2} d s,
$$

where, on an interior edge $T^{\prime}=\partial T^{1} \cap \partial T^{2}$ of $T_{h}$, we set $M(v)=M_{v^{1}}(v)=M_{v^{2}}(v)$, and, on a boundary edge $T^{\prime}$ of $T_{h}$, we set $M(v)=M_{\nu}(v)$. Then we define $V_{h}$ to be the completion of $\stackrel{V}{V}_{h}$ with respect to $\|\mathbf{v}\|_{0, h}$. It is clear that

$$
\|v\|_{0, h} \leqslant\left(\sum_{i, j}\left\|v_{i j}\right\|_{0, h}^{2}\right)^{1 / 2}
$$

for all $\mathbf{v} \in H^{1}(\Omega)=\left\{\mathbf{v}=\left(v_{i j}\right), 1 \leqslant i, j \leqslant 2: v_{12}=v_{21}, v_{i j} \in H^{1}(\Omega)\right\}$. When we use the norm $\|\cdot\|_{0, h}$, it will be clear from the context whether we are applying it to scalarvalued or matrix-valued functions. As in Subsection (a) we let $W_{h}=H_{h}^{2} \cap H_{0}^{1}$. Then the mixed method studied in this subsection is based on the following formulation of (4.1):

Given $g \in H^{-1}(\Omega)$, find $(u, \psi) \in V_{h} \times W_{h}$ satisfying

$$
\left\{\begin{array}{c}
\sum_{i, j=1}^{2} \int_{\Omega} u_{i j} v_{i j} d x+\sum_{i, j=1}^{2} \sum_{T \in T_{h}}-\int_{T} v_{i j} \frac{\partial^{2} \psi}{\partial x_{i} \partial x_{j}} d x \\
+\int_{\Gamma_{h}} M(\mathbf{v}) J \frac{\partial \psi}{\partial \nu} d s=0 \quad \forall \mathbf{v} \in V_{h} \\
\sum_{i, j=1}^{2} \sum_{T \in T_{h}}-\int_{T} u_{i j} \frac{\partial^{2} \varphi}{\partial x_{i} \partial x_{j}} d x+\int_{\Gamma_{h}} M(\mathrm{u}) J \frac{\partial \varphi}{\partial \nu} d s=-\int_{\Omega} g \varphi d x \quad \forall \varphi \in W_{h} .
\end{array}\right.
$$

Using (4.29), we can easily establish the relations between (4.1) and (4.31). If $\psi$ is the solution of (4.1) and $u_{i j}=\partial^{2} \psi / \partial x_{i} \partial x_{j}$, then $(u, \psi)$ is a solution of (4.31), and if $(\mathrm{u}, \psi)$ is a solution of (4.31), then $\psi$ is the solution of (4.1) and $u_{i j}=\partial^{2} \psi / \partial x_{i} \partial x_{j}$.

(4.31) is an example of problem $P$ with $V_{h}$ and $W_{h}$ as above,

and

$$
a_{h}(\mathbf{u}, \mathbf{v})=\sum_{i, j} \int_{\Omega} u_{i j} v_{i j} d x
$$

$$
b_{h}(\mathbf{u}, \varphi)=\sum_{i, j} \sum_{T}-\int_{T} u_{i j} \frac{\partial^{2} \varphi}{\partial x_{i} \partial x_{j}} d x+\int_{T_{h}} M(\mathbf{u}) J \frac{\partial \varphi}{\partial \nu} d s .
$$

Letting $S_{h}$ be as defined in (3.1), we consider the approximate problem $\mathrm{P}_{h}$ with

$$
V_{h}=\left\{\mathbf{v}=\left(v_{i j}\right): v_{12}=v_{21}, v_{i j} \in S_{h}\right\}
$$

and

$$
W_{h}=S_{h} \cap H_{0}^{1}(\Omega)
$$


With this choice for the forms $a_{h}$ and $b_{h}$ and spaces $V_{h}$ and $W_{h}$, problem $\mathrm{P}_{h}$ describes the Herrmann-Miyoshi method [15], [16] , [20]. Note that with this method we obtain direct approximations to $\psi$ and $\partial^{2} \psi / \partial x_{i} \partial x_{j}$ (the displacement and moments in elasticity problems).

In order to apply Theorem 1, we must check (2.1), (2.2), (2.5), and (2.6). (2.1) and (2.2) are immediate. In light of (4.30), the proof of (2.5) is similar to the proof of (2.5) for the method in Subsection (a). Finally we consider (2.6). Let $\varphi \in W_{h}$ be given. By Theorem 3 we know there is a $v \in S_{h}$ such that

$$
\int_{\Omega} \nabla_{v} \cdot \nabla \varphi d x \geqslant\|\varphi\|_{2, h}^{2}
$$

and

$$
\|v\|_{0, h} \leqslant C\left\|_{\varphi}\right\|_{2, h}
$$

Now let $\mathbf{v}=\left(\begin{array}{ll}v & 0 \\ 0 & v\end{array}\right)$. We immediately have $\mathbf{v} \in W_{h}$,

and

$$
b_{h}(\mathbf{V}, \varphi)=\sum_{i, j} \int_{\Omega} \frac{\partial v_{i j}}{\partial x_{j}} \frac{\partial \varphi}{\partial x_{i}} d x=\int_{\Omega} \nabla v \cdot \nabla \varphi d x \geqslant\|\varphi\|_{2, h}^{2},
$$

$$
\|\mathrm{v}\|_{0, h} \leqslant\left(\sum_{i, j}\left\|v_{i j}\right\|_{0, h}^{2}\right)^{1 / 2} \leqslant \sqrt{2} C\left\|_{\varphi}\right\|_{2, h} .
$$

This proves (2.6).

We are now ready to apply Theorem 1 to analyze the Herrmann-Miyoshi method. This application is essentially the same as that in Subsection (a). We use the approximability results in Lemma 3, as modified for matrix-valued functions with the aid of (4.30), and in Lemma 4. We will just state the results.

Suppose $\psi \in H^{r}(\Omega), r \geqslant 3$. Then

$$
\left\|\mathbf{u}-\mathbf{u}_{h}\right\|_{0, h}+\left\|\psi-\psi_{h}\right\|_{2, h} \leqslant C h^{s-2}\|\psi\|_{s}
$$

and

$$
\left\|\psi-\psi_{h}\right\|_{1} \leqslant C h^{s-1}\|\psi\|_{s},
$$

where $s=\min (r, k+1)$. From (4.32) we obtain

$$
\left\|\mathbf{u}-\mathbf{u}_{h}\right\|_{0} \leqslant C h^{s-2}\|\psi\|_{s} .
$$

Estimates (4.33) and (4.34) improve on those in Brezzi-Raviart [7]. Rannacher [22] recently obtained these estimates for the case $k=2$. Falk-Osborn [12] also proved these estimates. We further note that (4.32) contains additional information corresponding to the mesh dependent norms; cf. (4.25).

(c) Herrmann-Johnson Method. In this subsection we consider a further method for the approximate solution of (4.1) in which, as in the case treated in Subsection (b), the auxiliary variable is the matrix of second-order partials of $\psi$. Also as in Subsection (b), the method is based on the variational formulation (4.31) (the spaces $V_{h}$ and $w_{h}$ and the forms $a_{h}$ and $b_{h}$ are the same as in Subsection (b)). 
We now consider the problem $\mathrm{P}_{h}$ with

$$
V_{h}=\left\{\mathbf{v} \in \stackrel{\circ}{V}_{h}:\left.v_{i j}\right|_{T} \in P_{k-1} \forall T \in T_{h}\right\}
$$

and

$$
W_{h}=S_{h} \cap H_{0}^{1}(\Omega) \text {. }
$$

This choice leads to the Herrmann-Johnson method [15], [16], [17]. Note that this method differs from the Herrmann-Miyoshi method only in the choice of the finitedimensional space $V_{h}$.

This example has certain special features which allow an analysis that is rather different than that employed in the previous two examples. These special features involve the existence of two particular projection operators denoted by $\pi_{h}$ and $\Sigma_{h}$. We turn to this now.

$\pi_{h}$ is defined as in [7, Section 4]. For $\mathbf{v}=\left(v_{i j}\right)$ with $v_{i j} \in H^{1}(T)$ and $v_{12}=$ $v_{21}$, we define $\pi_{T} \mathbf{v}=\left(w_{i j}\right)$ with $w_{i j} \in P_{k-1}$ and $w_{12}=w_{21}$ by

$$
\begin{cases}\int_{T^{\prime}} M_{\nu}\left(\mathbf{v}-\pi_{T} \mathbf{v}\right) f d s=0 & \forall f \in P_{k-1} \text { and for each side } T^{\prime} \text { of } T, \\ \int_{T}\left[v_{i j}-\left(\pi_{T} \mathbf{v}\right)_{i j}\right] f d x=0 & \forall f \in P_{k-2} .\end{cases}
$$

By Lemma 3 in [6], $\Pi_{T} v$ is uniquely determined by (4.35). Now for $v \in \stackrel{\circ}{V}_{h}$, we define $\pi_{h} \mathbf{v} \in V_{h}$ by

$$
\left.\left(\pi_{h} \mathbf{v}\right)\right|_{T}=\pi_{T}\left(\left.\mathbf{v}\right|_{T}\right)
$$

Since we can write

$$
b_{h}(\mathbf{v}, \varphi)=\sum_{T \in T_{h}}\left\{-\sum_{i, j} \int_{T} v_{i j} \frac{\partial^{2} \varphi}{\partial x_{i} \partial x_{j}} d x+\int_{\partial T} M_{\nu}(\mathbf{v}) \frac{\partial \varphi}{\partial \nu} d s\right\}
$$

it is clear that

$$
b_{h}\left(\mathbf{v}-\pi_{h} \mathbf{v}, \varphi\right)=0 \quad \forall \varphi \in W_{h} .
$$

Concerning the approximation of $\mathbf{v}$ by $\pi_{h} \mathbf{v}$, we have

$$
\text { Lemma 6. Suppose } \mathrm{v} \in\left[H^{r-2}(\Omega)\right]^{4} \cap \stackrel{\circ}{V}_{h}, r \geqslant 3 \text {. Then }
$$

$$
\left\|\pi_{h} \mathbf{v}-\mathbf{v}\right\|_{0, h} \leqslant C h^{l}\left\|_{\mathbf{v}}\right\|_{l}
$$

for $1 \leqslant l \leqslant \min (k, r-2)$.

Proof. In Lemma 4 of [7] it is shown that

$$
\left\|\pi_{h} \mathbf{v}-\mathbf{v}\right\|_{0} \leqslant C h^{l}\left\|_{\mathbf{v}}\right\|_{l}
$$

Thus it remains to show that

$$
\left(h \int_{\Gamma_{h}}\left|M\left(\pi_{h} \mathrm{v}-\mathrm{v}\right)\right|^{2} d s\right)^{1 / 2} \leqslant C h^{l}\left\|_{\mathrm{v}}\right\|_{l} .
$$


Let $T \in T_{h}$ and assume $T$ is the image of $\hat{T}$ under the mapping $F(\hat{x})=B \hat{x}+b$. Given a matrix-valued function $\mathrm{w}(x)$ on $T$, we set $\hat{\mathrm{w}}(\hat{x})=C \mathrm{w}(F(\hat{x})) C^{t}, \hat{x} \in \hat{T}$, where $C=B^{-1}$. (Note that the correspondence between (matrix-valued) functions on $T$ and on $\hat{T}$ is different than the one introduced in Section 3.) Recall that $\nu=C^{t} \hat{\nu}\left|B^{t} \nu\right|$ ((3.5)). Then we have

$$
\begin{aligned}
& \int_{\partial T}\left|M_{\nu}\left(\mathbf{v}-\pi_{T} \mathbf{v}\right)\right|^{2} d s=\sum_{i} \int_{T_{i}^{\prime}}\left|M_{\nu}\left(\mathbf{v}-\pi_{T} \mathbf{v}\right)\right|^{2} d s=\sum_{i} \int_{T_{i}^{\prime}}\left|\nu^{t}\left(\mathbf{v}-\pi_{T} \mathbf{v}\right) \nu\right|^{2} d s \\
& =\sum_{i} \int_{T_{i}^{\prime}}\left|\hat{\nu}^{t} C B\left(\hat{\mathbf{v}}-\pi_{\hat{T}} \hat{v}\right)\left(F^{-1}(x)\right) B^{t} C^{t} \hat{\nu}\right|^{2}\left|B^{t} \nu\right|^{4} d s \\
& \leqslant\|B\|^{4} \max \left|T_{i}^{\prime}\right| \int_{\partial \hat{T}}\left|M_{\hat{\nu}}\left(\hat{\mathbf{v}}-\pi_{T} \hat{\mathbf{v}}\right)\right|^{2} d \hat{s} \leqslant C(\hat{T}) h_{T}\|B\|^{4}|\hat{\mathbf{v}}|_{l, T}^{2} \\
& \leqslant C(\hat{T}) h_{T}\|B\|^{2(l+2)}\|C\|^{4}|\operatorname{det} B|^{-1}|\mathbf{v}|_{l, T}^{2} \\
& \leqslant \frac{C(\hat{T}) h_{T}^{4}|\hat{T}| 4 \sigma^{6}}{\rho_{\hat{T}}^{2(l+2)} \pi} h^{2 l-1}|\mathbf{v}|_{l, T}^{2} .
\end{aligned}
$$

Hence

$$
h \int_{\Gamma_{h}}\left|M\left(\mathbf{v}-\pi_{h} \mathbf{v}\right)\right|^{2} d s \leqslant \sum_{T} h \int_{\partial T}\left|M_{\nu}\left(\mathbf{v}-\pi_{T} \mathbf{v}\right)\right|^{2} d s \leqslant C h^{2 l}|v|_{l, \Omega}^{2} .
$$

This completes the proof.

The second projection operator $\Sigma_{h}$ is the interpolation operator $I_{h}$ introduced in Section 3. As in the proof of Lemma 5 in [7], for $\mathbf{v} \in \stackrel{\circ}{V}_{h}$ and $\varphi \in H^{2}(\Omega) \cap$ $H_{0}^{1}(\Omega)$, we can write

$$
\begin{aligned}
b_{h}(\mathbf{v}, \varphi)= & -\sum_{T} \sum_{i, j} \int_{T} \frac{\partial^{2} v_{i j}}{\partial x_{i} \partial x_{j}} \varphi d x+\sum_{T^{\prime} \in I_{h}} \int_{T^{\prime}} A\left(T^{\prime}, \mathbf{v}\right) \varphi d s \\
& +\sum_{a \in J_{h}} B(a, \mathbf{v}) \varphi(a),
\end{aligned}
$$

where $I_{h}$ is the set of all sides of the triangulation $T_{h}, J_{h}$ is the set of all vertices of $T_{h}$, and $A\left(T^{\prime}, \mathbf{v}\right)$ is a polynomial of degree less than or equal to $k-2$ in the variable s. Since for $\mathbf{v} \in V_{h}$ we have $\partial^{2} v_{i j} /\left.\partial x_{i} \partial x_{j}\right|_{T} \in P_{k-3}$ and $A\left(T^{\prime}, \mathbf{v}\right) \in P_{k-2}$, it follows from (4.38) that $\Sigma_{h} \varphi=I_{h} \varphi$, as defined in Section 3, satisfies

$$
b_{h}\left(\mathbf{v}, \Sigma_{h} \varphi-\varphi\right)=0 \quad \forall \mathbf{v} \in V_{h} .
$$

Now we are ready to derive the error estimates. First we estimate $\left\|\mathbf{u}-\mathbf{u}_{\boldsymbol{h}}\right\|_{0}$. Subtracting (2.4a) from (2.3a), we obtain

$$
a_{h}\left(\mathbf{u}-\mathbf{u}_{h}, \mathbf{v}\right)+b_{h}\left(\mathbf{v}, \psi-\psi_{h}\right)=0 \quad \forall \mathbf{v} \in V_{h} .
$$

Suppose $\mathbf{v} \in Z_{h} \equiv\left\{\mathrm{w} \in V_{h}: b_{h}(\mathrm{w}, \varphi)=0 \forall \varphi \in W_{h}\right\}$. Then from (4.39) we see that $b_{h}(\mathrm{v}, \varphi)=b_{h}\left(\mathrm{v}, \Sigma_{h} \varphi\right)=0$ for all $\varphi \in H^{2}(\Omega) \cap H_{0}^{1}(\Omega)$. Hence from (4.40) we have

$$
a_{h}\left(\mathbf{u}-\mathbf{u}_{h}, \mathbf{v}\right)=0 \quad \forall \mathbf{v} \in Z_{h} .
$$


Subtracting (2.4b) from (2.3b) and using (4.36), we see that

$$
b_{h}\left(\pi_{h} \mathbf{u}-\mathbf{u}_{h}, \varphi\right)=b_{h}\left(\mathbf{u}-\mathbf{u}_{h}, \varphi\right)=0 \quad \forall \varphi \in W_{h},
$$

i.e., $\pi_{h} \mathbf{u}-\mathbf{u}_{h} \in Z_{h}$. Thus, recalling (4.41),

$$
\begin{aligned}
\left\|\mathbf{u}-\mathbf{u}_{h}\right\|_{0}^{2} & =a_{h}\left(\mathbf{u}-\mathbf{u}_{h}, \mathbf{u}-\mathbf{u}_{h}\right) \\
& =a_{h}\left(\mathbf{u}-\mathbf{u}_{h},\left(\mathbf{u}-\pi_{h} \mathbf{u}_{h}\right)+\left(\pi_{h} \mathbf{u}-\mathbf{u}_{h}\right)\right) \\
& =a_{h}\left(\mathbf{u}-\mathbf{u}_{h}, \mathbf{u}-\pi_{h} \mathbf{u}\right) \leqslant\left\|\mathbf{u}-\mathbf{u}_{h}\right\|_{0}\left\|\mathbf{u}-\pi_{h} \mathbf{u}\right\|_{0}
\end{aligned}
$$

and hence

$$
\left\|\mathbf{u}-\mathbf{u}_{h}\right\|_{0} \leqslant\left\|\pi_{h} \mathbf{u}-\mathbf{u}\right\|_{0} .
$$

Suppose now that $\psi \in H^{r}(\Omega), r \geqslant 3$. Then from (4.42) and Lemma 6 we have

$$
\left\|\mathbf{u}-\mathbf{u}_{h}\right\|_{0} \leqslant C h^{s}\|\psi\|_{s+2},
$$

where $s=\min (k, r-2)$.

Now we estimate $\psi-\psi_{h}$. As in Subsection (a) we can write

$$
\left(d, \psi-\psi_{h}\right)_{0}=-a_{h}\left(\mathbf{u}-\mathbf{u}_{h}, \mathbf{w}-\mathbf{z}\right)-b_{h}\left(\mathbf{w}-\mathbf{z}, \psi-\psi_{h}\right)
$$

$$
-b_{h}\left(\mathbf{u}-\mathbf{u}_{h}, \theta-\mu\right) \quad \forall(\mathbf{z}, \mu) \in V_{h} \times W_{h},
$$

where $\theta$ is the solution of

$$
\begin{cases}\Delta^{2} \theta=d \in L_{2} & \text { on } \Omega, \\ \theta=\frac{\partial \theta}{\partial \nu}=0 & \text { on } \Gamma\end{cases}
$$

and $w_{i j}=\partial^{2} \theta / \partial x_{i} \partial x_{j}$. We note that $(\mathbf{w}, \theta)$ satisfies

$$
\begin{cases}a_{h}(\mathbf{w}, \mathbf{v})+b_{h}(\mathrm{v}, \theta)=0 & \forall \mathrm{v} \in V_{h}, \\ b_{h}(\mathrm{w}, \varphi)=-\int_{\Omega} d \varphi d x & \forall \varphi \in W_{h}\end{cases}
$$

(cf. (4.31)). In (4.44) let $\mathrm{z}=\pi_{h} \mathrm{w}$ and $\mu=\Sigma_{h} \theta$. This gives

$$
\left(d, \psi-\psi_{h}\right)_{0}=-a_{h}\left(\mathbf{u}-\mathbf{u}_{h}, \mathbf{w}-\pi_{h} \mathbf{w}\right)-b_{h}\left(\mathbf{w}-\pi_{h} \mathbf{w}, \psi-\psi_{h}\right)
$$

$$
-b_{h}\left(\mathbf{u}-\mathbf{u}_{h}, \theta-\Sigma_{h} \theta\right)
$$

We now estimate each term in (4.46).

Using (4.36), (4.39), (4.45), and Lemma 3, we have

$$
\begin{aligned}
\left|b_{h}\left(\mathrm{w}-\pi_{h} \mathrm{w}, \psi-\psi_{h}\right)\right| & =\left|b_{h}\left(\mathrm{w}-\pi_{h} \mathrm{w}, \psi-\Sigma_{h} \psi\right)\right| \\
& =\left|b_{n}\left(\mathrm{w}, \psi-\Sigma_{h} \psi\right)\right|=\left|\left(d, \psi-\Sigma_{h} \psi\right)_{0}\right| \\
& \leqslant\|d\|_{0}\left\|\psi-\Sigma_{h} \psi\right\|_{0} \leqslant C h^{s}\|\psi\|_{s}\|d\|_{0},
\end{aligned}
$$

where $s=\min (r-1, k+1)$. 
In our estimate for the third term on the right side of (4.46), we treat the cases $k \geqslant 2$ and $k=1$ separately. First assume $k \geqslant 2$. Then, using (4.26), (4.39), and Lemmas 4 and 6 , we find that

$$
\begin{aligned}
\left|b_{h}\left(\mathbf{u}-\mathbf{u}_{h}, \theta-\Sigma_{h} \theta\right)\right| & =\left|b_{h}\left(\mathbf{u}-\pi_{h} \mathbf{u}, \theta-\Sigma_{h} \theta\right)\right| \\
& \leqslant C\left\|_{\mathbf{u}}-\pi_{h} \mathbf{u}\right\|_{0, h}\left\|\theta-\Sigma_{h} \theta\right\|_{2, h} \leqslant C h^{s-1}\left\|_{\mathbf{u}}\right\|_{s-1} h\|\theta\|_{3} \\
& \leqslant C h^{s}\|\psi\|_{s+1}\|d\|_{0},
\end{aligned}
$$

where $s=\min (r-1, k+1)$. Now suppose $k=1$. Then, using (4.26), (4.31), (4.39), and Lemma 3 , we have

$$
\begin{aligned}
\left|b_{h}\left(\mathbf{u}-\mathbf{u}_{h}, \theta-\Sigma_{h} \theta\right)\right| & =\left|b_{h}\left(\mathbf{u}, \theta-\Sigma_{h} \theta\right)\right|=\left|\left(\Delta^{2} \psi, \theta-\Sigma_{h} \theta\right)_{0}\right| \\
& \leqslant\left\|\Delta^{2} \psi\right\|_{0}\left\|\theta-\Sigma_{h} \theta\right\|_{0} \leqslant C h^{2}\left\|\Delta^{2} \psi\right\|_{0}\|\theta\|_{2} \\
& \leqslant C h^{2}\|\psi\|_{4}\|d\|_{0} .
\end{aligned}
$$

Finally, using (4.26), (4.42), and Lemma 6, we obtain

$$
\left|a_{h}\left(\mathbf{u}-\mathbf{u}_{h}, \mathbf{w}-\pi_{h} \mathbf{w}\right)\right| \leqslant\left\|\mathbf{u}-\mathbf{u}_{h}\right\|_{0}\left\|\mathbf{w}-\pi_{h} \mathbf{w}\right\|_{0} \leqslant C h^{s}\|\psi\|_{s+1}\|d\|_{0},
$$

where $s=\min (k+1, r-1)$.

Combining (4.46)-(4.50), we have

$$
\begin{aligned}
\left\|\psi-\psi_{h}\right\|_{0} & =\sup _{d \in L_{2}} \frac{\left|\left(d, \psi-\psi_{h}\right)_{0}\right|}{\|d\|_{0}} \\
& \leqslant C h^{s}\|\psi\|_{s+1}, \quad s=\min (k+1, r-1), \text { if } k \geqslant 2
\end{aligned}
$$

and

$$
\left\|\psi-\psi_{h}\right\|_{0} \leqslant C h^{2}\|\psi\|_{4}, \quad \text { if } k=1 .
$$

One can also prove that

$$
\left\|\psi-\psi_{h}\right\|_{1} \leqslant C h^{s-1}\|\psi\|_{s}, \quad s=\min (r, k+1), \text { if } k \geqslant 2
$$

and

$$
\left\|\psi-\psi_{h}\right\|_{1} \leqslant C h\|\psi\|_{3}, \quad \text { if } k=1 .
$$

Estimate (4.53) improves on estimates in [7]. Estimates (4.43) and (4.51)-(4.53) are given in [7], and (4.43) and (4.51)-(4.54) are proved in [12].

Remarks. (1) As in Subsection (b), we could have shown that the method studied here is stable with respect to the norm \|\|$_{0, h}+\|\|_{2, h}$ and then obtained error estimates in this norm. This approach would have allowed the treatment of the case when $g \in\left(H_{h}^{2}\right)^{\prime}-H^{-1}(\Omega)$ (cf. the next to the last paragraph in Subsection (a)). However, due to the special nature of this example, more refined estimates can be obtained by the analysis sketched above in the case when sufficient regularity of the solution is assumed. Thus the mesh dependent norms play a less central role in the analysis of 
this method than in previous methods. They are, however, convenient; their use leads to a very natural setting for the study of this example.

(2) The analysis in this subsection was based on the projections $\pi_{h}$ and $\Sigma_{h}$ and the fact that

$$
Z_{h} \subset Z \equiv\left\{\mathbf{w}: \mathbf{w} \in V_{h}, b_{h}(\mathbf{w}, \varphi)=0 \forall \varphi \in H^{2}(\Omega) \cap H_{0}^{1}(\Omega)\right\},
$$

which follows from the existence of $\Sigma_{h}$. For a general discussion of the projections $\pi_{h}$ and $\Sigma_{h}$ and the condition $Z_{h} \subset Z$ see Falk and Osborn [12] and Fortin [13].

(3) In this subsection the mesh family is not required to be quasi-uniform.

Acknowledgement. The second author would like to thank R. Falk for several helpful discussions on mixed methods.

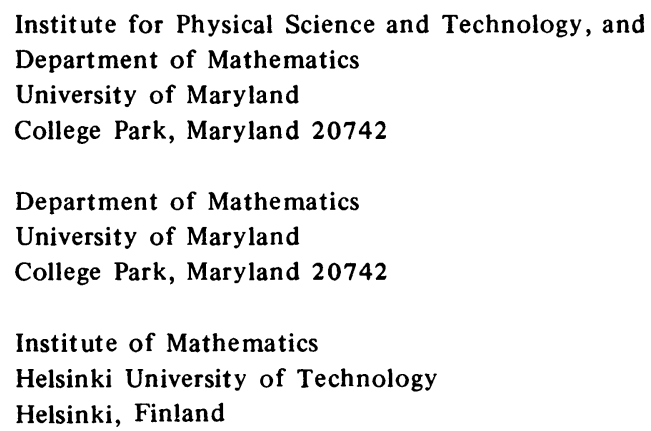

1. I. BABUŠKA, "Error-bounds for finite element method," Numer. Math., v. 16, 1971, pp. $322-333$.

2. I. BABUŠKA \& A. AZIZ, "Survey lectures on the mathematical foundations of the finite element method" in The Mathematical Foundations of the Finite Element Method with Application to Partial Differential Equations (A. K. Aziz, Ed.), Academic Press, New York, 1973, pp. 5-359.

3. I. BABUŠKA \& J. OSBORN, "Analysis of finite element methods for second order boundary value problems using mesh dependent norms," MRC Tech. Summary Report \#1919, University of Wisconsin-Madison; Numer. Math., v. 34, 1980, pp. 41-62.

4. J. BRAMBLE \& S. HILBERT, "Estimation of linear functionals on Sobolev spaces with application to Fourier transforms and spline interpolation," SIAM J. Numer. Anal., v. 13, 1976, pp. 185-197.

5. F. BREZZI, "On the existence, uniqueness and approximation of saddle-point problems arising from Lagrangian multipliers," R.A.I.R.O., R2, v. 8, 1974, pp. 129-151.

6. F. BREZZI, "Sur la méthode des éléments finis hybrides pour le problème biharmonique," Numer. Math., v. 24, 1975, pp. 103-131.

7. F. BREZZI \& P. RAVIART, "Mixed finite element methods for 4th order elliptic equations," Topics in Numerical Analysis III (J. Miller, Ed.), Academic Press, New York, 1978.

8. P. CIARLET, The Finite Element Method for Elliptic Problems, North-Holland, Amsterdam, 1978.

9. P. CIARLET \& P. RAVIART, "A mixed finite element method for the biharmonic equation," Symposium on Mathematical Aspects of Finite Elements in Partial Differential Equations (C. de Boor, Ed.), Academic Press, New York, 1974, pp. 125-143.

10. P. CLEMENT, "Approximation by finite element functions using local regularization," R.A.I.R.O., R2, 1975, pp. 77-84.

11. J. DOUGLAS, JR. \& T. DUPONT, Interior Penalty Procedures for Elliptic and Parabolic Galerkin Methods, Lecture Notes in Phys., No. 58, Springer-Verlag, Berlin, 1976.

12. R. FALK \& J. OSBORN, Error Estimates for Mixed Methods, MRC Tech. Summary Report \#1936, University of Wisconsin-Madison, 1979.

13. M. FORTIN, "Analysis of the convergence of mixed finite element methods," R.A.I.R.O., v. 11, 1977, pp. 341-354. 
14. R. GLOWINSKI, “Approximations externes par éléments finis de Lagrange d'ordre un et deux, du problème de Dirichlet pour l'opérateur biharmonique. Méthodes iteratives de résolutions des problèms approchés, in Topics in Numerical Analysis (J. J. H. Miller, Ed.), Academic Press, New York, 1973, pp. 123-171.

15. L. HERRMANN, "Finite element bending analysis for plates," J. Eng. Mech., Div. ASCE EM5, v. 93, 1967, pp. 49-83.

16. L. HERRMANN, "A bending analysis for plates," Proc. Conf. on Matrix Methods in Structural Mechanics, AFFDL-TR-66-88, pp. 577-604.

17. C. JOHNSON, "On the convergence of a mixed finite element method for plate bending problems," Numer. Math., v. 21, 1973, pp. 43-62.

18. R. KELLOGG \& J. OSBORN, “A regularity result for the Stokes problem," J. Funct. Anal., v. 21, 1976, pp. 397-431.

19. B. MERCIER, "Numerical solution of the biharmonic problems by mixed finite elements of class $C^{0}$," Boll. Un. Mat. Ital., v. 10, 1974, pp. 133-149.

20. T. MIYOSHI, "A finite element method for the solution of fourth order partial differential equations," Kunamoto J. Sci. (Math.), v. 9, 1973, pp. 87-116.

21, J. OSBORN, "Analysis of mixed methods using mesh dependent spaces," Computational Methods in Nonlinear Mechanics (J. T. Oden, Ed.), North-Holland, Amsterdam, 1980.

22. R. RANNACHER, "On nonconforming and mixed finite element methods for plate bending problems-the linear case." (Preprint.)

23. R. SCHOLZ, "Approximation von Sattelpunkten mit Finiten Elementen," Tagungsband, Bonn. Math. Schr., v. 89, 1976, pp. 53-66.

24. R. SCHOLZ, "A mixed method for 4 th order problems using linear finite elements," R.A.I.R.O., v. 12, 1978, pp. 85-90.

25. R. SCHOLZ, "Interior error estimates for a mixed finite element method." (Preprint.)

26. J. THOMAS, Sur l'Analyse Numérique des Méthodes d'Eléments Finis Hybrides et Mixtes, Thesis, Université P \& M Curie, Paris, 1977. 